\title{
Servo Turret Modal Analysis based on Finite Element Method
}

\author{
Zhe $\mathrm{Li}^{1, \mathrm{a}}$, Nan Chen ${ }^{1, \mathrm{~b}}$ and Ning Zhang ${ }^{1, \mathrm{c}}$ \\ ${ }^{1}$ School of Mechanical Engineering, Southeast University, Nanjing 211189 \\ azliseu@163.com, bnchen@seu.edu.cn, cnzhang_cn@seu.edu.cn
}

\begin{abstract}
Keywords: Servo turret; Finite element method; Modal analysis; Virtual material; Equivalent contact area

Abstract: Firstly, using CAD software SolidWorks to establish a geometric model of the servo turret. Then, using HyperMesh to pretreat the model. In the established finite element model the equivalent contact area method was used to simulate the bolt connection. At the same time, the virtual material method was used to equivalent bearing constraint. The equivalent contact area method effectively solve the nonlinear problems and improve the accuracy of bolt modeling. And the method of virtual material equivalent bearing constraints, the characteristics of the bearing was reflected more comprehensively. These two methods improve the quality of the finite element model and make the calculation results more accurately. Finally import the model to Abaqus for modal calculations. The first six natural frequencies of the servo CNC turret and its corresponding modal shapes are obtained in Abaqus. The results show that the servo turret has an adequate overall dynamic stiffness and no resonance phenomenon occurs. The modal analysis results provide theoretical basis for further harmonic response analysis and experimental modal tests. It can also indicate the direction of further improvement of the turret.
\end{abstract}

\section{Introduction}

As the core functional compenent of $\mathrm{CNC}$ machine tools, the performance of the servo CNC turret is an important criterion for judging the technical level of the machine tool being equipped, which has a major impact on the overall performance of the machine tool. The main function of the servo CNC turret is that the drive system is driven by the motor to finish the transposition of the cutterhead, so that the tool installed on the cutterhead realizes the work position conversion, shortens the auxiliary time in the machining process such as tool change and thus improves the workpiece ratio. Servo CNC turret is a relatively complex interdisciplinary system. With the continuous development of technology, domestic and foreign scholars have also done a lot of research. Burger, MJ [1] found that when the tool carrier is used for high-speed cutting, if carbide tools are used the vibration generated will greatly affect the processing and product precision. Xie Liming et al. [2] established the dynamic model of the turret structure in the machining center by using the mechanical impedance condensation method, analyzed its dynamic characteristics, and found out the weak links of the system. And make improvements. Wang Taiyong [3] studied the dynamic characteristics of lathe tool-turret system to find ways to improve cutting performance and reduce noise and vibration.

This article first establishes the three-dimensional model of the CNC turret in SolidWorks, then imports the model into HyperMesh for finite element pretreatmenting, and finally imports the finite element model into Abaqus for modal calculation. By analyzing the natural frequency and natural modal shapes of the servo CNC turret and judging its dynamic characteristics, the research results provide a theoretical basis for the next harmonic response analysis and experimental modal test of the $\mathrm{CNC}$ turret, and indicate the direction of the $\mathrm{CNC}$ turret structure improvement work.

\section{Main structure of the servo CNC turret}

The main mechanical structure of the servo CNC turret is shown in the figure below. The main components are the cutter head, triple gear plate structure (internal gear plate, external gear plate, right gear plate), shaft gear, pinion unit, piston, hydraulic cylinder head and electric switch, etc. . The pinion unit drives the internal gear plate to realize the transposition of the cutter head and achieves the 
target position to perform rough positioning. The hydraulic system drives the piston to drive the right gear plate to control the CNC turret to achieve the precise positioning of the cutter head. The servo CNC turret studied in this paper has 8 tool positions. The cutter head can rotate bidirectionally to achieve the nearest tool selection. It can withstand large cutting forces and has a wide range of applications.

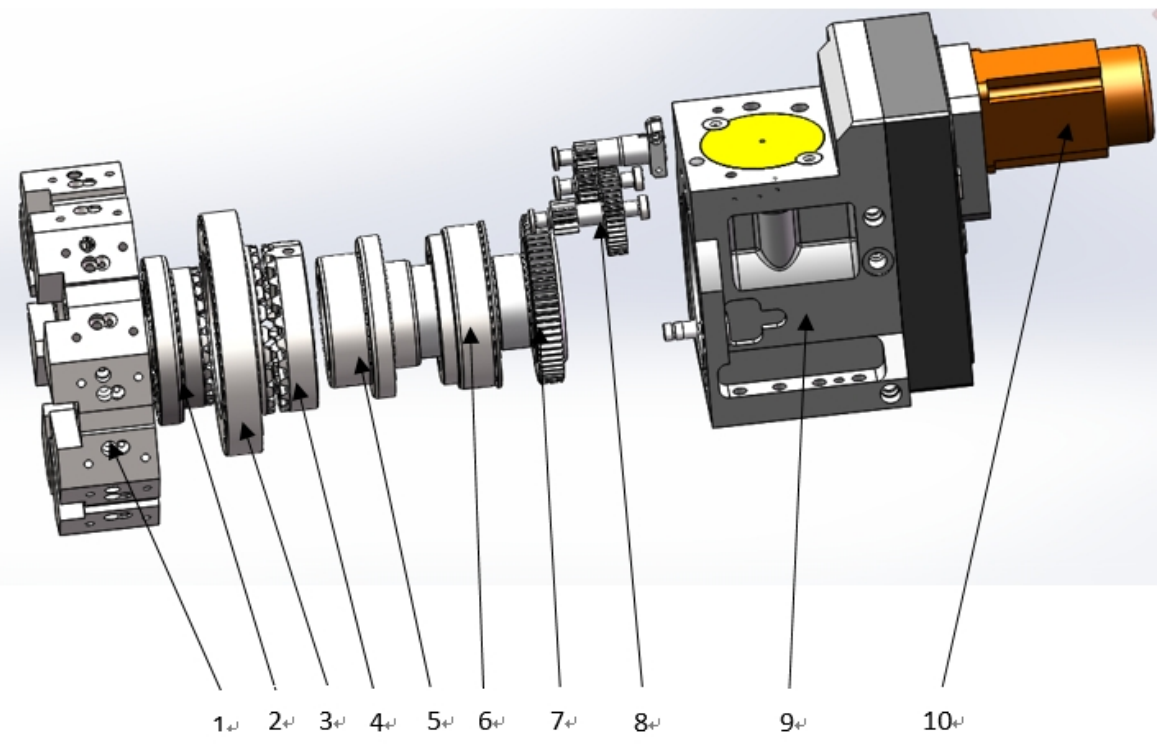

1. cutter head 2. internal gear plate, 3. external gear plate 4. right gear plate 5. piston 6. hydraulic cylinder head 7. shaft gear 8. pinion unit 9.casing 10.servo motor

Fig.1 Main structure of the servo CNC turret

\section{Modal analysis theory}

Modality is an inherent property of the mechanical structure [4]. Each mode corresponds to its natural frequency, natural modal shapes and modal damping. These inhered characteristics are important reference indicators for dynamic analysis. Modal analysis is the basic of dynamic analysis of mechanical structures. The above inhered properties can be obtained through experiments or finite element analysis, and then modal analysis can be performed. This is also the basic of harmonic response analysis and random vibration analysis.

For an n-degree-of-freedom linear vibration system, the differential equation of motion can be expressed as a matrix:

$$
[M]\{+[C]\{+[K]\{x\}=\{f(\mathrm{t})\}
$$

In the formula $[M],[C],[K]$ are respectively the system's mass matrix, damping matrix and stiffness matrix, and all are square matrices. For a linear vibration system, each coefficient matrix is usually a real coefficient symmetric matrix. $\{\{\{x\}$ are n-order arrays of acceleration, velocity, and displacement response, respectively. $\{f(\mathrm{t})\}$ is the n-order array of incentives.

Set incentive and response are

$$
\{f(\mathrm{t})\}=\{F\} e^{j \omega t}\{x(\mathrm{t})\}=\{X\} e^{j \omega t}
$$

Substitute (1), then 


$$
\left([K]-\omega^{2}[M]+j \omega[C]\right)\{X\}=\{F\}
$$

make

$$
[H(\omega)]^{-1}=[K]-\omega^{2}[M]+j \omega[C]
$$

If you abbreviate $[H(\omega)]^{-1}$ to $[H]^{-1}$, the formula above can be expressed as

$$
[H]^{-1}[X]=[F]
$$

In the formula, $[H]$ is called the transfer function matrix, which is also the square matrix of the order.

After a lot of previous calculation experience shows that the effect of damping on the natural frequency, modal shapes, etc. of the system can be almost negligible at this time (1) can be written as

$$
[M]\{+[K]\{x\}=\{f(\mathrm{t})\}
$$

For an undamped free vibration system, Equation (4) can be written as

$$
[M]\{\leftrightarrow+[K]\{x\}=0
$$

Let the solution of the above equation be

$$
\{x\}=\{X\} e^{j \omega t}
$$

Substituting the above equation into equation (5) can be solved

$$
\left([K]-\omega^{2}[M]\right)\{X\}=0
$$

The natural frequency of the system $\omega_{i}(i=1,2, \cdots, \mathrm{n})$ can be solved by the above equation, that is, the undamped natural frequency of the system is obtained. Substituting $\omega_{i}$ the above formula into the corresponding displacement vector $\{X\}$ can obtain the natural modal shapes of the system.

\section{Servo CNC turret modal analysis}

\section{Establishing three-dimensional model}

In the three-dimensional software SolidWorks, the three-dimensional model of the servo CNC turret is established, and then the model is imported into HyperMesh for pretreatmenting of the finite element calculation. In order to improve the quality of mesh division and improve the efficiency of finite element calculation, the model and its components are simplified to ignore the small features such as small holes, chamfers, and fillets in the 3D model and will have a greater impact on the dynamic characteristics of the CNC turret. Small parts are deleted. 


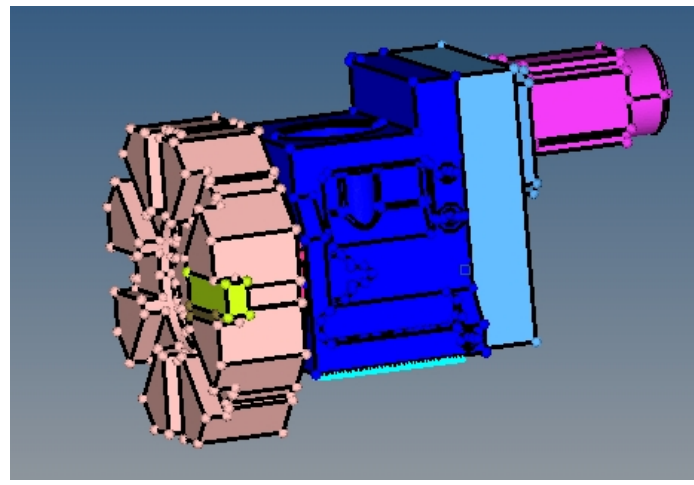

Fig2 finite element model of Servo turret

Inquired the model of the servo motor used in this article, converted the quality and volume of the query into the corresponding density of the motor to the corresponding material property of the motor; at the same time, the quality of the gear train and the main shaft gear was queried in the three-dimensional software. The weight of the pinion unit is added to the shaft gear, and the newly obtained shaft gear density is given to the material properties of the shaft gear.

Table 1 Material Properties of the Servo CNC turret

\begin{tabular}{|c|c|c|c|c|}
\hline Name & Material & $\operatorname{Density}\left(\mathrm{t} / \mathrm{mm}^{3}\right)$ & elasticity ( $\mathrm{MPa})$ & $\begin{array}{l}\text { Poisson's } \\
\text { ratio }\end{array}$ \\
\hline $\begin{array}{l}\text { cutter head v piston } \\
\text { hydraulic cylinder } \\
\text { head } \text { motor } \\
\text { connection board }\end{array}$ & 45 steel & $7.89 e^{-9}$ & $2.09 e^{5}$ & 0.269 \\
\hline $\begin{array}{l}\text { internal gear plate } \\
\text { external gear plate }\end{array}$ & 20CrMnTi & $7.86 e^{-9}$ & $2.07 e^{5}$ & 0.289 \\
\hline $\begin{array}{l}\text { right gear plate、 } \\
\text { adjustment gasket of } \\
\text { shaft gear }\end{array}$ & $40 \mathrm{Cr}$ & $7.87 e^{-9}$ & $2.11 e^{5}$ & 0.277 \\
\hline $\begin{array}{c}\text { casing } \begin{array}{c}\text { Rear cover of } \\
\text { casing }\end{array}\end{array}$ & QT550 & $7.02 e^{-9}$ & $1.62 e^{5}$ & 0.291 \\
\hline motor & self-defined1 & $7.80 e^{-9}$ & $2.09 e^{5}$ & 0.269 \\
\hline shaft gear & self-defined 2 & $12.5 e^{-9}$ & $2.12 e^{5}$ & 0.289 \\
\hline
\end{tabular}

\section{Equivalent treatment of bolt connection}

Nonlinear behaviors such as geometric nonlinearity, material nonlinearity, and contact nonlinearity, compared to static analysis, cannot be calculated by finite element modal analysis. Therefore, nonlinear behavior is treated as linear, that is, to maintain the initial State [5]. A large number of components are bolted in the servo CNC turret, but the bolt connection is a typical non-linear connection. In the case of contact, the stiffness matrix will change with the change of the contact conditions, so it is very important to define the connection area and the corresponding stiffness as accurately as possible in the finite element software.

In the past some bolt modeling, the bolt joint surface is often bonded together, which is actually inaccurate. According to the relevant experience in actual use, using two pieces of work piece fixed by bolts, under the effect of bolt pre-tightening force, only a part of the contact surface can be tightly connected, with the area closest to the bolt hole being the tightest, and the area far from the bolt hole. The connection is loose. The workpieces with bolted joints are mainly distributed in an approximately conical region under the action of pre-tightening force. Osgood's proposed range of envelope angles for this region is [6]. Then, as shown in Fig. 3, the areas with large stress can be treated in the finite 
element according to the bonding method. By analyzing the areas where the stress on the contact surface is greater, it can be found and bonded to the equivalent simulated bolted connection area, transforming the nonlinearity of the bolted connection into a linear problem [7]. The actual bonding area is related to the bolt size and connection depth. In this way, not only the nonlinear problem of bolt connection is solved, but also the effective area of the bolt connection is effectively equivalent, and the overall quality of the finite element model is improved.

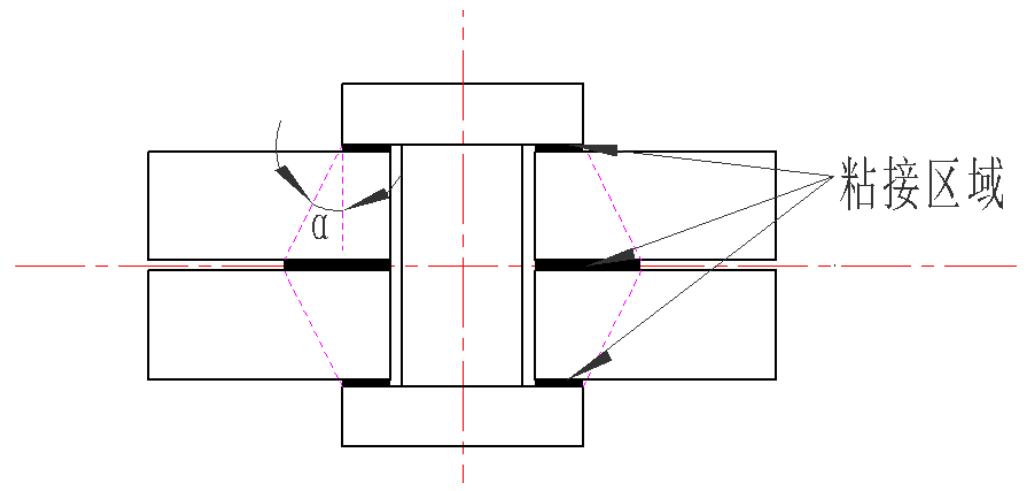

Fig. 3 Bonding area of the bolt equivalent model

\section{Treatment of Bearing Equivalent Constraints}

Shaft gear's one end of servo CNC turret studied in this paper is bolted to the internal gear plate, and the other end is matched with the radial needle roller bearing. In the process of finite element analysis, the equivalent handling of the needle bearing is simplified according to its bearing characteristics and connection form. In the past, the equivalent of the bearing unit simulation mostly used a series of spring units in order to reflect the important characteristics stiffness of the bearing in the model and the other characteristics of the bearing could not be reflected. The virtual material method can simulate more characteristics of the model material. A series of characteristics such as quantity, Poisson's ratio, and material density can reflect the characteristics of the bearing more comprehensively and improve the accuracy of the model as a whole. Therefore, the virtual material method is used here to make bearing connection equivalent.

The method of the virtual material simulates the mechanical properties of the connecting surface by changing the elastic modulus, Poisson's ratio, and density of the virtual material. This method is a virtual material method [8]. The properties of the virtual material in the modal analysis are the elastic modulus E, Poisson's ratio and density. These properties can be calculated using the theoretical formula as follows [9].

$$
\begin{aligned}
& E\left(\mathrm{~A}_{r}>\mathrm{A}_{r c}\right)=\frac{2}{3 \pi^{2}} D \psi^{1-0.5 D} E G^{D-1} a_{l}^{0.5 D}\left(a_{c}^{-0.5}-a_{l}^{-0.5}\right) \\
& \mu=\frac{\left(1-\mu^{\prime}\right) \mathrm{E}^{*}}{G_{x}^{*}}-1 \\
& \rho=\frac{\rho_{1} l_{1}+\rho_{2} l_{2}}{l_{1}+l_{2}}
\end{aligned}
$$

In the formula: $D$ - Fractal dimension; $\psi$ - Parameters determined by the fractal dimension; $E^{\prime}$ Equivalent elastic modulus; $G$ - Fractal feature length dimension; $a_{l}$ - Microbump maximum contact area; $a_{c}-$ Microbump critical contact Area: $\mu^{\prime}-$ Equivalent Poisson's ratio; $E^{*}-$ Dimensionless 
virtual material elastic modulus; $G_{x}^{*}$ - Dimensionless virtual shear modulus;- $\rho_{1} 、 \rho_{2}$ - Density of two mating surface materials; $l_{1} 、 l_{2}$ - The thickness of the two bodies.

The formulas for the key parameters in equations (7), (8) and (9) are as follows ${ }^{[9]}$.

$$
\begin{aligned}
& \frac{1}{E^{\prime}}=\frac{1-\mu_{2}^{2}}{E_{1}}+\frac{1-\mu_{2}^{2}}{E_{2}} \\
& a_{l}=\frac{2-D}{D} \psi^{1-0.5 D} A_{r} \\
& a_{c}=\frac{G^{2}}{(0.5 \mathrm{~K} \phi)^{\frac{2}{D-1}}} \\
& \mu^{\prime}=\frac{E^{\prime}}{2 G^{\prime}}-1 \\
& E^{*}=\frac{E}{E^{\prime}} \\
& G^{\prime}=\frac{2-\mu_{1}}{G_{1}}+\frac{2-\mu_{2}}{G_{2}}
\end{aligned}
$$

In the formula: $E_{1} 、 E_{2} 、 \mu_{1} 、 \mu_{2}$ - the elastic modulus and Poisson's ratio of the two contact materials; $A_{r}$ - the actual contact area; $K=\frac{H}{\sigma_{y}}$ - the ratio of the hardness and the yield limit of the softer material; $\phi=\frac{\sigma_{y}}{E^{\prime}}$ - the yield strength and the equivalent elasticity of the softer material Modulus ratio; $G_{x}^{*}$ - Equivalent shear modulus; $G_{1} 、 G_{2}$-Shear modulus of two contact materials.

After the corresponding calculation of the relevant values of the bearing virtual material is shown in the following table.

Table 2 Bearing Virtual Material Properties

\begin{tabular}{ccccc}
\hline Name & Material & Density $\left(\mathrm{t} / \mathrm{mm}^{3}\right)$ & elasticity $(\mathrm{MPa})$ & $\begin{array}{l}\text { Poisson's } \\
\text { ratio }\end{array}$ \\
\hline Bearing & $\begin{array}{c}\text { Virtual } \\
\text { material }\end{array}$ & $5.43 e^{-9}$ & $1.908 e^{7}$ & 0.38 \\
\hline
\end{tabular}

\section{Solving in Abaqus}

Save the pretreatmented model in HyperMesh as an inp format file, open it in Abaqus and create a job, submit the calculation, and complete the modal simulation solution. Viewing the modal simulation results of the tool rest in the software, you can see the natural frequency and vibration pattern of the CNC turret.

The first six-order modal shapes of the tool post are shown in the figure below 


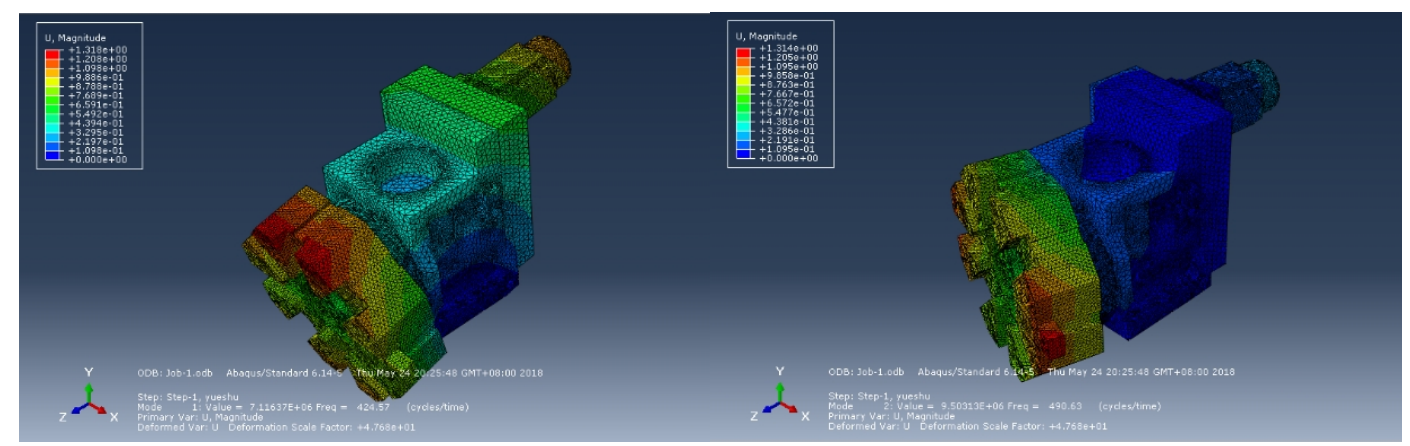

(a) The first and second modal shape

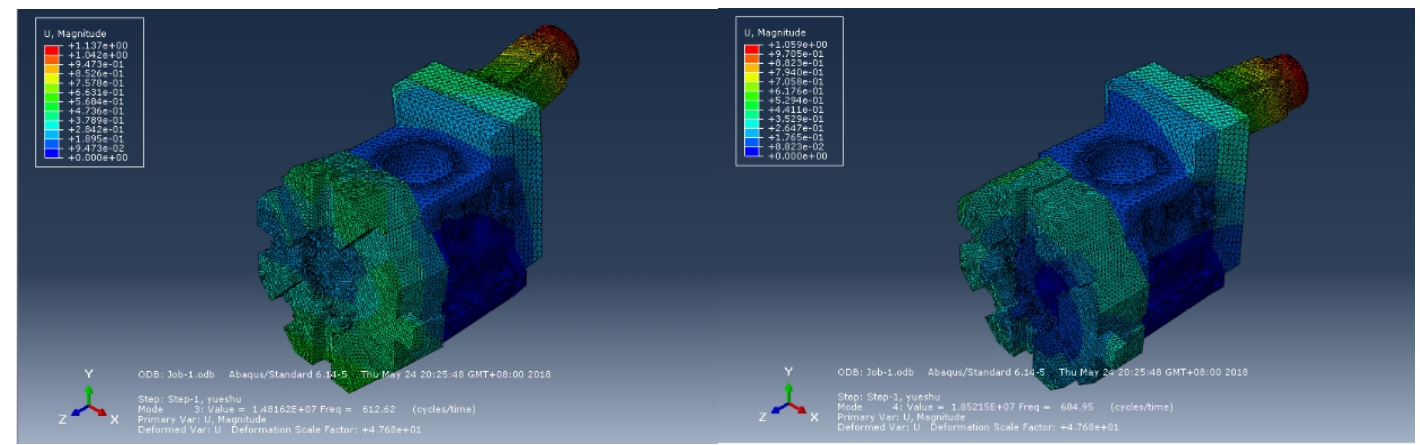

(b) The third and fourth modal shape

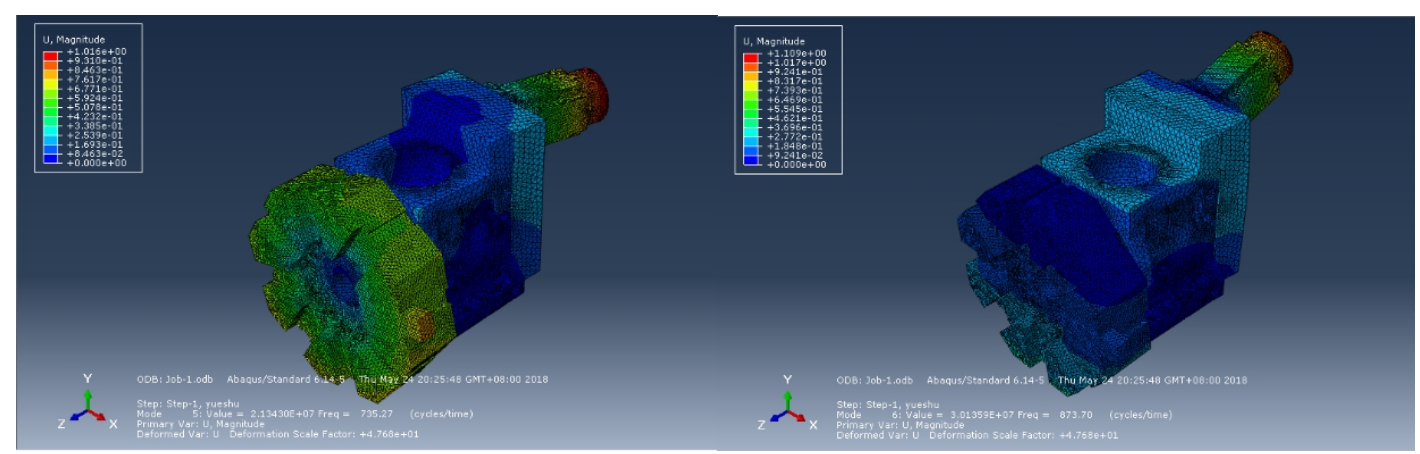

(c) The fifth and sixth modal shape

Figure 4 modal analysis of the first six-order modal shapes

The first six natural frequencies and modes of the $\mathrm{CNC}$ turret are shown in the following table

Table 3 The first six-order natural frequency and its corresponding mode

\begin{tabular}{ccc}
\hline Order & Natural frequency / $(\mathrm{Hz})$ & The description of the mode \\
\hline 1 & 424.57 & The cutter head nodded downwards \\
2 & 490.63 & The cutter head rotates along y-axis \\
3 & 612.62 & Cutter head, motor squeezed into the middle of the casing \\
4 & 684.95 & Cutter head rotates clockwise along Z axis \\
5 & 735.27 & Cutter rotates counterclockwise along Z axis \\
6 & 873.70 & Upward deflection of the motor \\
\hline
\end{tabular}

According to the analysis results of the above table, the first order frequency of the CNC turret is 424.57 Hz. From the relevant experience of the machine tool, the speed of the spindle of the machine tool generally does not exceed $6000 \mathrm{r} / \mathrm{min}$, and thus the cutting excitation of the machine tool is less 
than or equal to $100 \mathrm{~Hz}$. Therefore, the first-order natural frequency of the $\mathrm{CNC}$ turret is much larger than the machine's cutting excitation. It can be seen that the dynamic stiffness of the CNC turret is very good, which can effectively avoid the resonance phenomenon.

The equivalent contact area method improve the accuracy of bolt modeling. And the method of virtual material equivalent bearing constraints, the characteristics of the bearing was reflected more comprehensively. The two methods make the calculation results more accurately

With reference to the magnitude of the first six natural frequencies of the $\mathrm{CNC}$ turret, the natural frequency of the harmonic response analysis of the CNC turret and the frequency bandwidth of the experimental modal analysis can be determined to be $0-1000 \mathrm{~Hz}$.

According to the analysis of the first six-order modal shapes of the CNC turret, it can be seen that the 1-5 modal shapes mainly reflect the movement of the cutter-head relative to the casing body, which also reflects that the joint between the cutter-head and the casing body is the weak part of the turret. The relatively weak link of the machine is a place that needs special attention in the further improvement of the CNC turret.

\section{Conclusion}

In this article the geometric model of CNC turret was established in SolidWorks. The model was pretreated in HyperMesh, and finally was imported into Abaqus for modal calculation. The first six-order natural frequencies and the corresponding modal shapes of the tool carrier were obtained.

(1) By analyzing the first six natural frequencies of the servo CNC turret, it shows that the natural frequency of the CNC turret is much higher than the cutting excitation frequency of the machine tool, which can effectively avoid the resonance phenomenon.

(2) The modal analysis results of the servo CNC turret lays the foundation for the subsequent harmonic response analysis, random vibration analysis and modal experiment.

(3) By analyzing the modal shapes of the CNC turret, the weak link of the CNC turret is pointed out and the direction for the further improvement of the CNC turret structure is indicated as well.

\section{Acknowledgements}

The research was supported by "National Science and Technology Major Project (2013ZX04012-032).

\section{References}

[1] Burger, M.J. Redesigining turret lathe for cemented carbide tools [J]. American Machinist, 1994(78): 732-733.

[2] Xie Liming, Li Guohui, Zha Fusheng. Dynamics modeling of milling and turning machining center [J]. Machine Tools \& Hydraulics, 2005(6): 62-63.

[3] Wang Taiyong, Zhang Jisuo. Research on dynamic performance of lathe-tool system [J]. Vibration and Noise Control, 1991(4): 10-12.

[4] Lin XJ. Identification of vibration modal parameters and its application [M]. Nanjing: Southeast University Press, 1994, 123-132.

[5] Gao Xu, Zeng Guoying, Li Tingting. Modal Analysis of Threaded Flange Structure Based on ANSYS [J]. Journal of Chongqing University of Science and Technology: Natural Science Edition, 2009, 11(5): 145-147.

[6] Jeong, Kima, Joo-Cheol, Yoon, Beom-Soo Kang. Finite element analysis and modeling of structure with bolted joints [I]. Applied Mathematical Modelling, 2007, 31: 895-911. 
[7] Cheng Lai, Song Yanming, Li He, Yang Yang. Modal Analysis of Bolted Joints Based on Equivalent Contact Region [J]. Mechanical Design \& Research, 2012, 28(2): 44-47.

[8] Huang Kaili, Jin Jianxin. Research on simulation of bolt preload based on virtual material method [J]. Mechanical Design \& Manufacture, 2012, 8: 148-150.

[9] TIAN Hong-liang, LIU Hong-qi, LI Bing. A new method of virtual material hypothesis-based dynamic modeling on fixed joint interface in machine tools [J].International Journal of Machine Tools \& Manufacture, 2011, 51(3): 239-249. 